\title{
Power of Moran's I Test for Spatial Dependence in Panel Data Models with Time Varying Spatial Weights Matrices
}

\author{
Bianling OU \\ School of Management Science and Engineering, Central University of Finance and Economics, \\ Beijing 102206, China \\ E-mail: oubianling@amss.ac.cn \\ Xin ZHAO \\ School of Management Science and Engineering, Central University of Finance and Economics, \\ Beijing 100081, China \\ E-mail: zhaoxin0515@126.com \\ Mingxi WANG \\ School of International Trade and Economics, University of International Business and Economics, \\ Beijing 100029, China \\ E-mail: chinesewang@163.com
}

\begin{abstract}
The spatial weights matrix is usually specified to be time invariant. However, when it are constructed with economic/socioeconomic distance, trade/demographic/climatic characteristics, these characteristics might be changing over time, and then the spatial weights matrix substantially varies over time. This paper focuses on power of Moran's I test for spatial dependence in panel data models with where spatial weights matrices can be time varying (TV-Moran). Compared with Moran's I test with time invariant spatial weights matrices (TI-Moran), the empirical power of TV-Moran test for spatial dependence are evaluated. Our extensive Monte Carlo simulation results indicate that Moran's I test with misspecified time invariant spatial weights matrices is questionable; Instead, TV-Moran test has shown superiority in higher power, especially for cases with negative spatial correlation parameters and the large time dimension.
\end{abstract}

Keywords time varying spatial weights matrices; Moran's I; spatial dependence; panel data models; Monte Carlo simulations

\section{Introduction}

As an important tool in economics, spatial econometric models are widely used in applied geographic and regional science studies, and have recently also been applied in empirical finance $^{[1-4]}$. Spatial econometric methods have been rapidly developed. Recently, time varying spatial dependence is closed concerned. Generally, it includes two kinds: Time varying spatial parameters and time varying spatial weights matrices. This paper focuses on the latter.

Received February 5, 2015, accepted March 17, 2015

This research was financially supported by the National Natural Science Foundation of China (71101143) 
Druska and Horrace ${ }^{[5]}$ estimated a panel data model where disturbances were spatially correlated in the cross-sectional dimension, time varying spatial parameter and weights matrix were also allowed by generalized moment methods. Kuethe et al. ${ }^{[6]}$ combined existing spatial econometric techniques with a model that allowed parameters to evolve over time, and captured both the temporal and spatial dynamics of housing prices. Rambaldi and Rao ${ }^{[7]}$ developed a class of models with time varying hedonic coefficients and spatially correlated errors, constructed and compared the corresponding price index series, and furthermore the time varying parameter with spatial errors were found to be the best model. Lee and $\mathrm{Yu}^{[8]}$ first investigated the quasi-maximum likelihood estimation of spatial dynamic panel data models where spatial weights matrices could be time varying, and found that QML estimate was consistent and asymptotically normal, however when spatial weights matrices substantially varied over time, a model misspecification of a time invariant spatial weights matrix might cause substantial bias in estimation. Hazir and Bernard ${ }^{[9]}$ worked on a spatial Durbin model, which included time varying spatial weights matrices, space and time lagged variables. Blasques et al. ${ }^{[10]}$ introduced a new model for time-varying spatial dependence, adopted the model to empirically investigate spatial dependence between eight European sovereign CDS spreads over the period 2009-2014. Brännäs ${ }^{[11]}$ considered a novel way of incorporating spatial weights matrix for both time and space dynamic models with or without simultaneity, and studied count data models corresponding to previously spatial econometric models for continuous variables.

As we know, the spatial weights matrix is an important characteristic element of spatial econometric models and is cause of dispute in relation to what is it and how should it be specified $^{[12]}$. It's mainly two steps for model estimation and tests for spatial dependence in spatial econometric models. However, there are a few of literatures about tests for spatial dependence with time varying spatial weights matrices so far. $\mathrm{Ou}^{[13]}$ first investigated Moran's I test for spatial dependence in panel data models where spatial weights matrices can be time varying, and Monte Carlo results indicate that based on time varying and misspecified time invariant spatial weights matrices, size of Moran's I test has no significant difference, and power of Moran's I test for spatial dependence with time varying spatial weights matrices is much higher.

The main aim of this paper is to develop tests for spatial dependence in panel data models, and further investigates the effectiveness of TV-Moran for different alternative hypothesis of spatial dependence, for different cross-sectional dimensions and for different time dimensions from the perspective of power. Based on extensive Monte Carlo simulations, we find that Moran's I test with misspecified time invariant spatial weights matrices is questionable; Instead, TV-Moran test has shown superiority in higher power, especially for cases with negative spatial correlation parameters and the large time dimension.

This paper is organized as follows. Section 2 is devoted to a description of Moran's I test for spatial dependence in panel data models with time varying spatial weights matrices. Section3 presents the design of Monte Carlo experiments and results. The conclusions are given in Section 4. 


\section{TV-Moran}

The spatial autoregressive model (SAR) is a standard tool to analyze data with spatial correlation. The spatial error autoregressive model (SEAR) is also usually one kind of spatial econometric models. When spatial weights matrices are time invariant, SAR and SEAR models are respectively the following Equations (1) and (2):

$$
\begin{aligned}
& Y=\rho\left(I_{T} \otimes W\right) Y+X \beta+\varepsilon \\
& Y=X \beta+u, \quad u=\lambda\left(I_{T} \otimes W\right) u+\varepsilon
\end{aligned}
$$

When spatial weights matrices in panel data models are changed over time, Equation (1) becomes

$$
Y=\rho \widetilde{W} Y+X \beta+\varepsilon
$$

and Equation (2) becomes

$$
Y=X \beta+u, \quad u=\lambda \widetilde{W} u+\varepsilon
$$

where both $\rho$ and $\lambda$ are spatial correlation parameters, and the NTXNT time varying spatial weights matrix $\widetilde{W}$ is as follows:

$$
\widetilde{W}=\left(\widetilde{w}_{i j, t}\right)_{i, j=1,2, \cdots, N, t=1,2, \cdots, T}=\left[\begin{array}{ccccc}
\widetilde{W}_{1} & 0 & \cdots & 0 & 0 \\
0 & \widetilde{W}_{2} & \cdots & 0 & 0 \\
\vdots & \vdots & \vdots & \vdots & \vdots \\
0 & 0 & \cdots & \widetilde{W}_{T-1} & 0 \\
0 & 0 & \cdots & 0 & \widetilde{W}_{T}
\end{array}\right]
$$

and

$$
\widetilde{W}_{t}=\left[\begin{array}{cccc}
\widetilde{w}_{11, t} & \widetilde{w}_{12, t} & \cdots & \widetilde{w}_{1 N, t} \\
\widetilde{w}_{21, t} & \widetilde{w}_{22, t} & \cdots & \widetilde{w}_{2 N, t} \\
\vdots & \vdots & \vdots & \vdots \\
\widetilde{w}_{N 1, t} & \widetilde{w}_{N 2, t} & \cdots & \widetilde{w}_{N N, t}
\end{array}\right]
$$

where $\widetilde{W}_{t}(t=1,2, \cdots, T)$ is an $N \times N$ row-normalized spatial weights matrix with zero diagonal in the $t$-th period, and when spatial weights matrices are time invariant, the sub-matrix $\widetilde{W}_{1}=$ $\widetilde{W}_{2}=\cdots=\widetilde{W}_{t}$ and $\widetilde{W}=I_{T} \otimes W$.

Hypothesis tests for spatial dependence are a common practice for the identification and estimation of a spatial econometric model. Moran's I test is a simple and popular test for spatial dependence ${ }^{[14-20]}$. Therefore, when spatial weights matrices change over time, Moran's I tests for spatial dependence in panel data models become:

$$
I=\frac{e^{\prime} \widetilde{W} e}{e^{\prime} e}
$$

where the residual $e=\left(I_{N T}-X\left(X^{\prime} X\right)^{-1} X^{\prime}\right) Y=M Y$. In this case, the matrix $M$ is still a real symmetric idempotent matrix, and the spatial weights matrix $W$ is still row standardized, which is similar to the cross sectional case ${ }^{[15,16]}$. Tiefelsdorf ${ }^{[21]}$ derived approximate distribution 
of Moran's I by its moments when the disturbance vector $\varepsilon$ is an independent and identically distributed (i.i.d.) random variable. Furthermore, we can develop asymptotic distribution of Moran's I test from panel data models with time invariant spatial weights matrices to those with time varying spatial weights matrices. Moran's I statistic in Equation (5) is asymptotic normal distributed, and its' expectation and variance follow that:

$$
\begin{aligned}
E(I) & =\frac{\operatorname{trace}(M \widetilde{W})}{N T-k} \\
V(I) & =\frac{\operatorname{trace}\left(M \widetilde{W} M \widetilde{W}^{\prime}\right)+\operatorname{trace}(M \widetilde{W})^{2}+[\operatorname{trace}(M \widetilde{W})]^{2}}{(N T-k)(N T-k+2)}-[E(I)]^{2}
\end{aligned}
$$

Then we can obtain the standardized Moran's I statistic and its' distribution by using the z-transformation of Moran's I:

$$
Z=\frac{I-E(I)}{\sqrt{V(I)}} \stackrel{\text { Asy }}{\sim} N(0,1)
$$

When spatial weights matrices substantially vary over time, tests for spatial dependence may be bias, and Equation (6) should be reasonably used. In the next section, we further investigate the performance of Moran's I test for spatial dependence with time varying spatial weights matrices.

\section{Monte Carlo Simulation}

In this section, based on Moran's I test for spatial dependence in panel data models with time varying spatial weights matrices, power are used to evaluate the performance of the test when there is spatial dependence. Specifically, given spatial dependence, we expect that the test will show a large probability of rejecting the null hypothesis of spatial independence, namely the power of the test.

\subsection{Experimental Design}

Given the null hypothesis of spatial independence, the alternatives under consideration include a spatial autoregressive model (SAR) and a spatial error autoregressive model (SEAR). In order to study the power of Moran's I test, two alternative models are used to generate spatial dependence data with time varying spatial weights matrices. The SAR and SEAR models are respectively defined by: $Y=\rho \widetilde{W} Y+X \beta+\varepsilon$, and $Y=X \beta+u, u=\lambda \widetilde{W} u+\varepsilon$. $X$ is an NTX3 matrix, generated from the independent uniformly distribution in $[0,10] . \varepsilon$ is an NTX1 vector, generated from the independent standardized normal distribution. When the alternative hypothesis model (SAR) is true, $\rho=-0.8,-0.6, \cdots, 0.6,0.8$, and for another alternative hypothesis model (SEAR), $\lambda=-0.8,-0.6, \cdots, 0.6,0.8$. The significance level is 0.05. For time varying spatial weights matrices, we choose a random pattern. Any element of the $t$-th spatial weights matrix $W_{t}$ is randomly drawn from 0 and 1 . All these spatial weights matrices are row-normalized and zero diagonal. We also compare power of Moran's I test for spatial dependence with time varying spatial weights matrices (TV-Moran) and time invariant spatial weights matrices (TI-Moran). 1000 replications are carried out. 


\section{$3.2 \quad$ Results}

The power curves of TV-Moran and TI-Moran are compared for different alternative models, for different cross-sectional dimensions, and for different time dimensions.

We first present the simulation results for SAR and SEAR models with the cross-sectional dimension $N=25,49$, and 81 and the time dimension $T=5,10$ and 15. At 5\% nominal level of significance, Figures 1 and 2 plot the power curves for three different cross sectional dimensions and for three time dimensions of two spatial model specifications, respectively. Three rows in the graphs correspond to three cross sectional dimensions 25, 49, and 81. Three columns are for three different time dimensions 5, 10, and 15 under consideration. In each of the 3 by 3 grid of graphs, "TV" and "TI" denote the power curves of Moran's I test for spatial dependence with time varying and misspecified time invariant spatial weights matrices, respectively.

From Figures 1 and 2, at 5\% nominal level of significance, when the alternative model is truly the SAR model, power of TV-Moran is much higher than that of TI-Moran when spatial correlation is negative. When spatial correlation is positive, they become equivalent. Both cross sectional dimensions and the alternative models have no significant influence on power of TV-Moran and TI-Moran tests for spatial dependence. We observe that power of TV-Moran in the SAR model is slightly higher than that in the SEAR model.
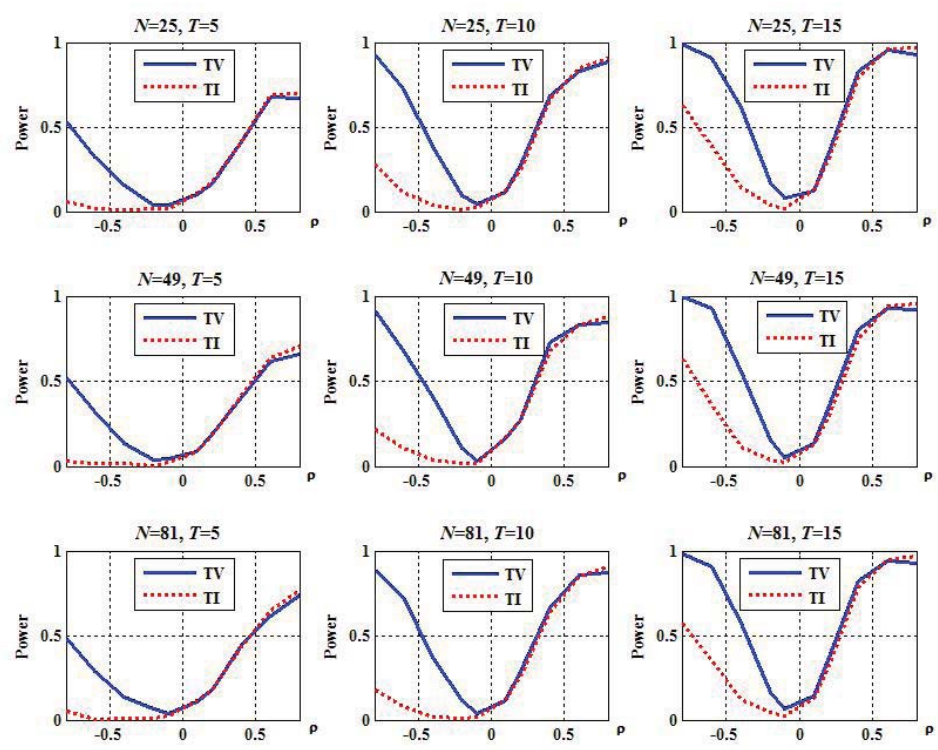

Figure 1 Power of Moran's I test for spatial dependence against the SAR model, for different spatial correlation parameters

Next given the spatial correlation parameter ( $\rho$ or $\lambda=-0.7,-0.3,0.3,0.7$ ), we consider power of TV-Moran and TI-Moran for different time dimensions. At $5 \%$ nominal level of significance, Figures 3 and 4 plot power curves for four spatial correlation parameters of two alternative models, respectively. From Figures 3 and 4, when spatial correlation is negative, the blue solid curve "TV" is much higher than the red dotted curve "TI". Specifically, when 
oor $\lambda=-0.3$, there becomes longer distance between the blue solid curve and the red dotted curve with larger time dimension; and however when $\rho$ or $\lambda=-0.7$, it's on the contrary.
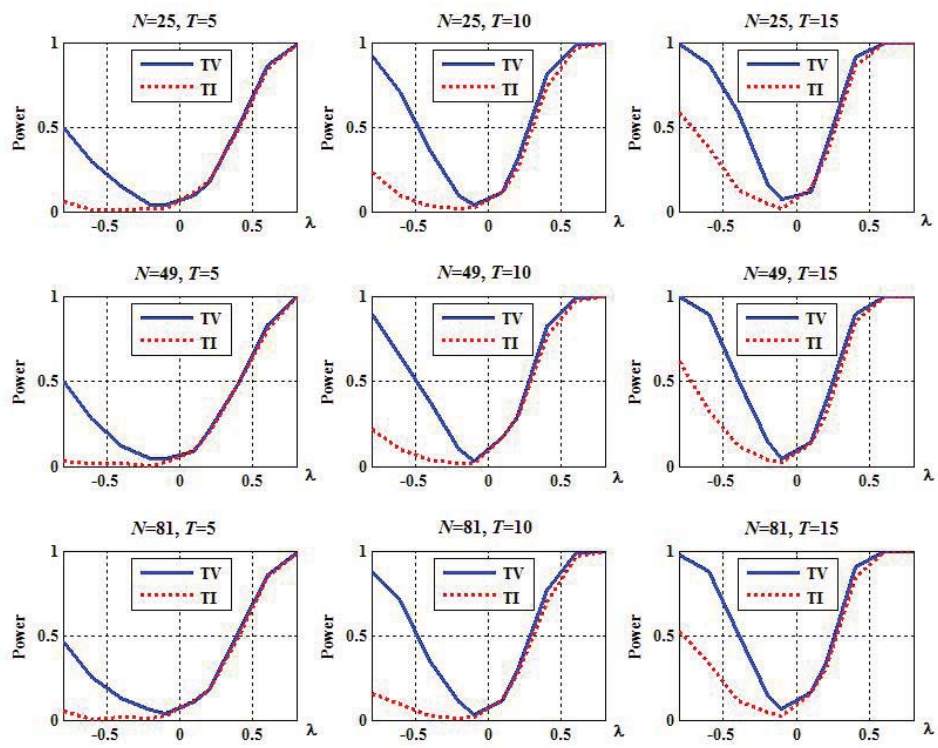

Figure 2 Power of Moran's I test for spatial dependence against the SEAR model, for different spatial correlation parameters
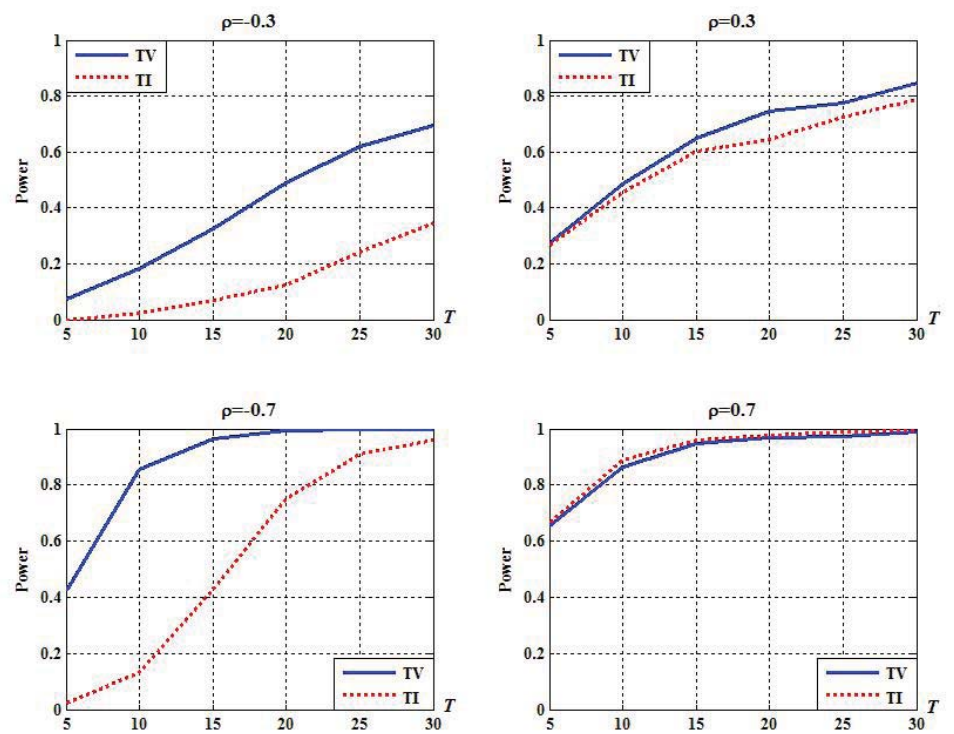

Figure 3 Power of Moran's I test for spatial dependence against the SAR model, for different time dimensions, given $N=49$

In a word, when spatial correlation is weak, power of TV-Moran is much higher than that 
of TI-Moran as time dimensions increase. For positive and strong spatial correlation, power of TV-Moran is slightly higher than that of TI-Moran.

Finally given spatial parameter ( $\rho$ or $\lambda=-0.7,-0.3,0.3,0.7$ ), we consider power of TVMoran and TI-Moran for different cross sectional dimensions. Figures 5 and 6 indicate that cross sectional dimensions have no significant influence on power. Specifically, when spatial correlation is negative, power of TI-Moran is much less than 0.1 , and however power of TVMoran is much higher than that of TI-Moran, especially $\rho$ or $\lambda=-0.7$. When spatial correlation is positive, power of TV-Moran is similar to that of TI-Moran.
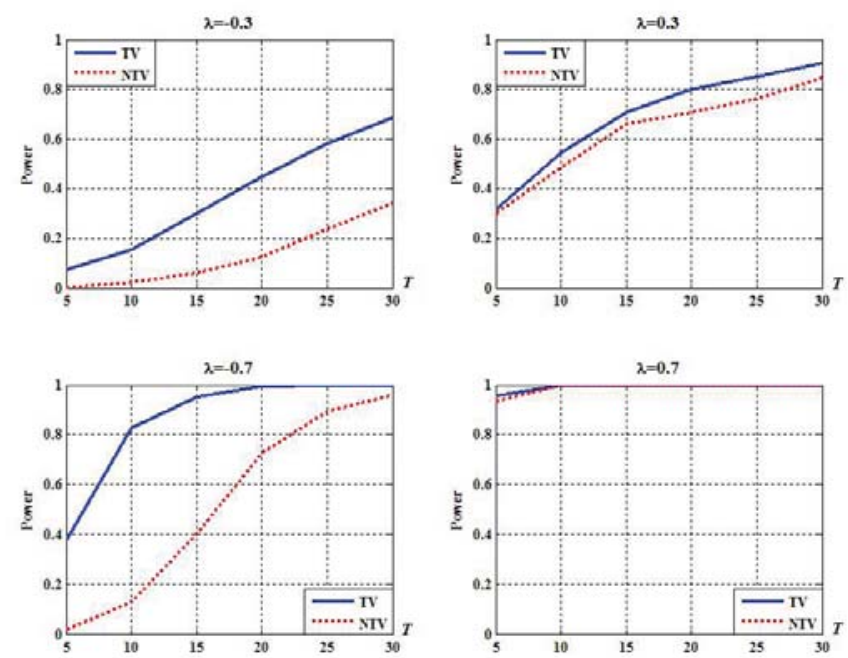

Figure 4 Power of Moran's I test for spatial dependence against the SEAR model, for different time dimensions, given $N=49$
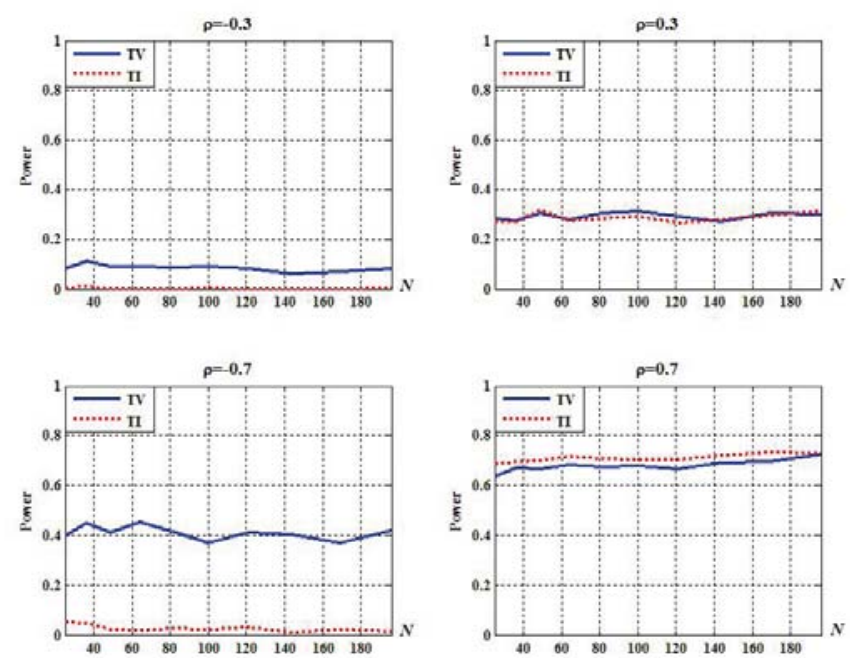

Figure 5 Power of Moran's I test for spatial dependence against the SAR model, for different cross-sectional dimensions, given $T=10$ 
In summary, based on our extensive Monte Carlo experiments, when there is negative spatial correlation, Moran's I test for spatial dependence with misspecified time invariant spatial weights matrices may cause substantial reduction in power, and TV-Moran is proven to have much higher power than TI-Moran.
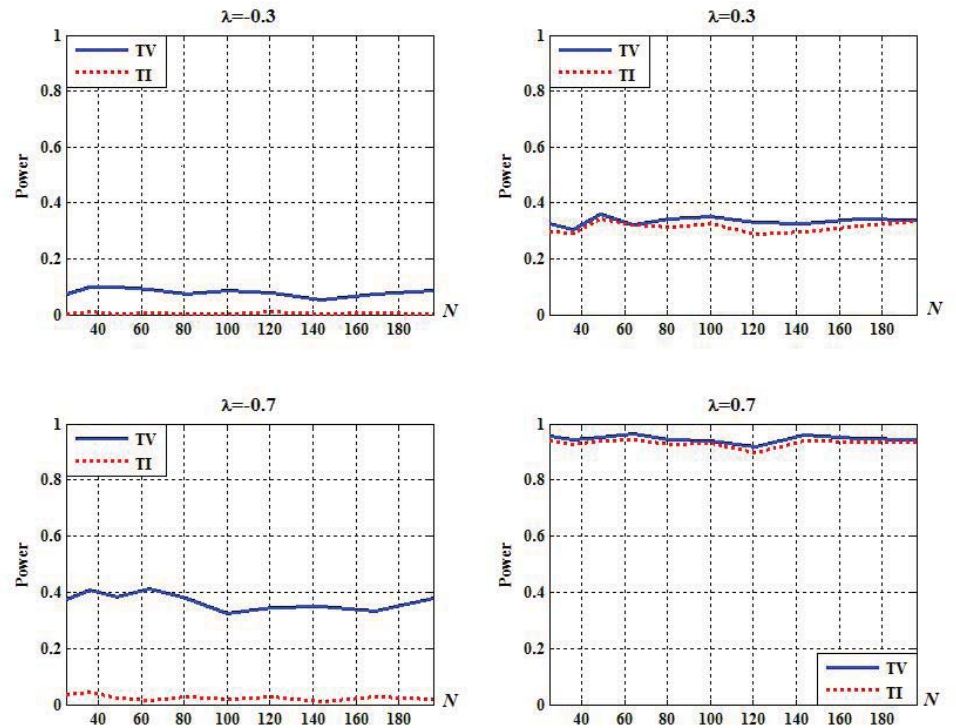

Figure 6 Power of Moran's I test for spatial dependence against the SEAR model, for different cross-sectional dimensions, given $T=10$

\section{Conclusion}

In this paper, we further investigate and develop Moran's I tests for spatial dependence in panel data with substantially time varying spatial weights matrices. It's known that TIMoran with misspecified time invariant spatial weights matrices performs poorly with weak power when spatial correlation is negative. Our Monte Carlo experiments confirm that with time varying spatial weights matrices, Moran's I test is superior to that with misspecified time invariant spatial weights matrices in the view of power. Specifically, when spatial correlation is negative, power of TV-Moran is much higher than that of TI-Moran. The time dimension is larger, and the power of TV-Moran is higher. Nevertheless, the alternative models and cross sectional dimensions have no significant influence on power of TV-Moran.

\section{References}

[1] Fernandez V. Spatial linkages in international financial markets. Quantitative Finance, 2011, 11: $237-245$.

[2] Wied D. Cusum-type testing for changing parameters in a spatial autoregressive model for stock returns. Journal of Time Series Analysis, 2013, 34: 221-229.

[3] Arnold M, Stahlberg S, Wied D. Modeling different kinds of spatial dependence in stock returns. Empirical Economics, 2013, 44: 761-774.

[4] Asgharian H, Hess W, Liu L. A spatial analysis of international stock market linkages. Journal of Banking and Finance, 2013, 37: 4738-4754.

[5] Druska V, Horrace W C. Generalized moments estimation for spatial panel data: Indonesian rice farming. American Journal of Agricultural Economics, 2004, 86(1): 185-198. 
[6] Kuethe T H, Foster K A, Florax R J G M. A spatial hedonic model with time-varying parameters: A new method using flexible least squares. Working Paper, 2008.

[7] Rambaldi A N, Rao D S P. Hedonic predicted house price indices using time-varying hedonic models with spatial autocorrelation. Working Paper, 2011.

[8] Lee L F, Yu J H. QML estimation of spatial dynamic panel data models with time varying spatial weights matrices. Spatial Economic Analysis, 2012, 7(1): 31-74.

[9] Hazir C S, Bernard C A. The effect of spatio-temporal knowledge flows on regional innovation performance: The case of ICT patenting in Europe. Working Paper, 2013.

[10] Blasques F, Koopman S J, Lucas A, et al. Spillover dynamics for systemic risk measurement using spatial financial time series models. Working Paper, 2014.

[11] Brännäs K. Adaptations of conventional spatial econometric models to count data. Working Paper, 2014.

[12] Mur J, Herrera M, Ruiz M. Selecting the W matrix: Parametric vs. nonparametric approaches. Working Paper, 2011.

[13] Ou B L. Moran's I tests for spatial dependence in panel data models with time varying spatial weights matrices. International Conference on Economic Management and Trade Cooperation (EMTC), Xi'an in China, 2014.

[14] Moran P. The interpretation of statistical maps. Journal of the Royal Statistical Society B, 1948, 10: $243-251$.

[15] Cliff A D, Ord J K. Spatial autocorrelation. Pion, London, 1973.

[16] Cliff A D, Ord J K. Spatial processes: Models and applications. Pion, London, 1981.

[17] Anselin L. Spatial econometrics: Methods and models. Kluwer Academic Publishers, Boston, 1988.

[18] Anselin L. Spatial externalities, spatial multipliers and spatial econometrics. International Regional Science Review, 2003, 26: 153-166.

[19] Anselin L, Bera A K, Florax R, et al. Simple diagnostic tests for spatial dependence. Regional Science and Urban Economics, 1996, 26: 77-104.

[20] Anselin L, Gallo J L, Jayet H. Spatial panel econometrics. Eds. by Matyas L, Sevestre P. The Econometrics of Panel Data, Springer-Verlag, Berlin, 2008.

[21] Tiefelsdorf M. Modeling spatial processes: The identification and analysis of spatial relationships in regression residuals by means of Moran's I. Spinger-Verlag, Berlin, 1999. 